\title{
Parameterized Macromodeling of Stochastic Linear Systems for Frequency- and Time-Domain Variability Analysis
}

\author{
Yinghao Ye*, Domenico Spina*, Giulio Antonini ${ }^{\dagger}$, and Tom Dhaene* \\ *IDLab, Department of Information Technology, Ghent University - imec, Gent, Belgium. \\ †UAq EMC Laboratory, Dipartimento di Ingegneria Industriale e dell'Informazione e di Economia, \\ Universitá degli Studi dell'Aquila, L'Aquila, Italy. \\ Email: yinghao.ye@ugent.be
}

\begin{abstract}
This paper presents a unique modeling framework able to describe general linear and passive systems depending on deterministic and stochastic parameters altogether. Once the stochastic macromodel is built, the variability analysis of the system under study can be accurately performed both in the frequency- and time-domain for any nominal value of the parameters considered in a suitable design space.
\end{abstract}

Index Terms-Polynomial Chaos, parameterized macromodeling, variability analysis, passivity.

\section{INTRODUCTION}

In the recent years, many Polynomial Chaos (PC) expansion based techniques, such as [1]-[6], have been proposed for uncertainty quantification applications in the electromagnetic and electronics domain, as alternative to the computationally cumbersome Monte Carlo (MC)-based approaches. In particular, these techniques focus on the efficient evaluation of the performance of the system considered, when some of its parameters can be considered as random variables, varying around a suitable and fixed nominal value.

In this contribution, we present an alternative approach applicable when the system performance must be evaluated for different nominal values of the geometrical or electrical parameters. Rather than focusing only on the fast computation of the stochastic variations, the proposed approach aims at giving a complete characterization both in the time- and frequency-domain of the system performance in the (large) range of variations of the possible nominal values of such parameters. The proposed approach is based on the stochastic modeling techniques [6] and it is described in the following.

\section{PARAmeterized Macromodeling of Polynomial Chaos-BAsed Augmented Systems}

In general, the scattering parameters of generic linear and passive systems $\boldsymbol{S}(s, \boldsymbol{g})$ depend on the frequency $s=j 2 \pi \omega$ and on a set of geometrical or electrical parameters $\boldsymbol{g}$. Typically, these design parameters can be assumed to have values in a suitable range, which can be discrete or continuous, and it will be referred to as design space in the rest of the contribution. During the design phase, the main goal is to find a set of values $\boldsymbol{g}=\boldsymbol{g}_{x}$ where the system under study satisfies the design specifications and is robust to the tolerances of the manufacturing process. In this framework, the geometrical or electrical design parameters under study can be considered as random variables with suitable distributions around their nominal values $\boldsymbol{g}$.

There are different possible approaches to reach this goal: for example, first a set of values in the design space (also called design points) satisfying the initial specifications can be chosen via suitable design of experiments (DoE) techniques $\left(\boldsymbol{g}_{1}, \boldsymbol{g}_{2}, \ldots, \boldsymbol{g}_{Q}\right)=\boldsymbol{G}$, then the variability analysis can be performed for all the elements of $G$, in order to verify which design points are robust to the tolerances of the manufacturing process. In this paper, we explore the possibility of computing a single parameterized macromodels describing the stochastic variations of the system under study both in the frequencyand time-domain in the entire design space of interest.

\section{A. PC-based Augmented Systems}

When a linear and passive system is subject to stochastic effects caused by random variations of its geometric or electrical parameters, here denoted by the random variables $\boldsymbol{\xi}$ varying around their nominal value $\boldsymbol{g}$, the relationship between the $\boldsymbol{\xi}$-dependent incident $\boldsymbol{a}(s, \boldsymbol{\xi} \mid \boldsymbol{g}) \in \mathbb{C}^{N \times 1}$ and reflected $\boldsymbol{b}(s, \boldsymbol{\xi} \mid \boldsymbol{g}) \in \mathbb{C}^{N \times 1}$ waves at the $N$ system ports becomes

$$
\boldsymbol{b}(s, \boldsymbol{\xi} \mid \boldsymbol{g})=\boldsymbol{S}(s, \boldsymbol{\xi} \mid \boldsymbol{g}) \boldsymbol{a}(s, \boldsymbol{\xi} \mid \boldsymbol{g})
$$

where $\boldsymbol{S}(s, \boldsymbol{\xi} \mid \boldsymbol{g})$ is the scattering matrix of the system under study. Following the approach described in [6], it is possible to define the following deterministic representation describing the variations of the system under study:

$$
\boldsymbol{b}_{P C}(s, \boldsymbol{g})=\boldsymbol{S}_{P C}(s, \boldsymbol{g}) \boldsymbol{a}_{P C}(s, \boldsymbol{g})
$$

where the vectors $\boldsymbol{a}_{P C}(s, \boldsymbol{g}), \boldsymbol{b}_{P C}(s, \boldsymbol{g}) \in \mathbb{C}^{(M+1) N \times 1}$ collects the deterministic PC expansion coefficients of the corresponding wave variables, with $M+1$ the number of the PC basis functions considered, whereas $\boldsymbol{S}_{P C}(s, \boldsymbol{g}) \in$ $\mathbb{C}^{(M+1) N \times(M+1) N}$ is a suitable scattering matrix representation, called "augmented system". 
It is important to remark that

- the augmented system is computed via a suitable weighted combination of the PC coefficients of the scattering matrix of the system under study;

- despite (2) is a mathematical description, it retains the physical properties of such system: $\boldsymbol{S}_{P C}(s, \boldsymbol{g})$ can be considered as an actual scattering parameters matrix which must follow the same constraints for causality, stability and passivity as for its original stochastic counterpart;

- computing such augmented system requires to evaluate the scattering parameters for a set of values $\boldsymbol{\xi}_{k}$ for $k=$ $1, \ldots, K$ of the chosen random parameters, in order to compute the corresponding $\mathrm{PC}$ expansion in the form

$$
\boldsymbol{S}(s, \boldsymbol{\xi} \mid \boldsymbol{g}) \approx \sum_{k=0}^{M} \boldsymbol{S}_{k}(s, \boldsymbol{g}) \varphi_{k}(\boldsymbol{\xi} \mid \boldsymbol{g})
$$

where $\varphi_{k}$ are the orthonormal PC basis functions and $\boldsymbol{S}_{k}(s, \boldsymbol{g})$ for $k=0, \ldots, M$ are the corresponding $\mathrm{PC}$ coefficients. Note that the PC basis functions are (typically) orthogonal polynomials and include a constant term, which is usually indicated with $\varphi_{0}$. When orthonormal basis functions are considered, as in this case, it is possible to prove that $\varphi_{0}=1$. The interested reader is referred to [1]-[6] for a detailed description of the PC expansion properties.

Now, since $\boldsymbol{S}_{P C}(s, \boldsymbol{g})$ can be considered as a scattering matrix, it is possible to employ any deterministic parameterized macromodeling technique defined for the scattering parameters [7]-[10] in order to model the stochastic variations of the system under study for all the possible nominal values $\boldsymbol{g}$ in the design space considered. Note that, while the timedomain variability analysis based on the augmented system (2) is described in [6], it is important to define also how such augmented system can be used for frequency-domain variability analysis.

Now, the $(i, j)$ block of $\boldsymbol{S}_{P C}(s, \boldsymbol{g})$ is defined as:

$$
\left[\boldsymbol{S}_{P C}(s, \boldsymbol{g})\right]_{i j}=\sum_{k=0}^{M} \boldsymbol{S}_{k}(s, \boldsymbol{g})\left\langle\varphi_{k}(\boldsymbol{\xi}) \varphi_{j}(\boldsymbol{\xi}), \varphi_{i}(\boldsymbol{\xi})\right\rangle
$$

where the symbol $\langle\cdot\rangle$ indicate the inner product operator. For $i=0$, equation (4) can be simplified as

$$
\left[\boldsymbol{S}_{P C}(s, \boldsymbol{g})\right]_{0 j}=\boldsymbol{S}_{j}(s, \boldsymbol{g})
$$

given the orthogonality of the PC basis functions and considering that $\varphi_{0}(\boldsymbol{\xi})=1$. Hence, the first block row of $\boldsymbol{S}_{P C}$ contains all the PC coefficients of the system scattering matrix. Similarly, by assuming $j=0$ in (4), the same conclusion can be drawn for the first block column. As a result, it is always possible to obtain the PC coefficients of the scattering matrix starting from its augmented system representation: the frequency-domain variability analysis can be performed with accuracy and efficiency thanks to the properties of the PC expansion. Furthermore, if a continuous frequency-domain model of $\boldsymbol{S}_{P C}$ is obtained, for example via the Vector Fitting
(VF) algorithm [11], [12], it is possible to evaluate the desired PC coefficients over the entire frequency range considered.

\section{B. Augmented Systems Parameterization}

At this point, it is possible to build a parameterized macromodel of $\boldsymbol{S}_{P C}$ and employ it for the time- and frequencydomain variability analysis of the system under study. In particular, we adopt the technique [7] together with the adaptive sampling scheme [13], in order to automate the model building process. The proposed modeling framework starts by computing the augmented system $\boldsymbol{S}_{P C}$ in the design points individuated by the technique [13]. In order to do so, a PC model of the scattering parameters in the form (3) is computed for each design point, starting from the scattering parameters of the system under study evaluated for a set of samples around the chosen design points [6]. Once the augmented systems $\boldsymbol{S}_{P C}$ are obtained, corresponding statespace models are computed via the VF algorithm (indicated here as root macromodels) and the parameterized model is computed through suitable interpolation schemes of such root macromodels [7]. If the modeling accuracy is not sufficient, the design space is divided in cells by the technique [13] and the entire procedure is repeated for each cell, until the desired accuracy is reached. The flowchart for this modeling process is illustrated in Fig. 1.

Hence, for each (nominal) value of the parameters $\boldsymbol{g}$ in the design space considered, the proposed modeling framework gives a state-space representation of the corresponding augmented system $\boldsymbol{S}_{P C}$, which can be used for both time- and frequency-domain analysis, as described in [6] and section II-A, respectively.

Furthermore, the proposed methodology allows one to model deterministic and stochastic parameters altogether: the parameters $\boldsymbol{g}$ in the design space can be deterministic, stochastic, or a combination of the two, as it will be shown

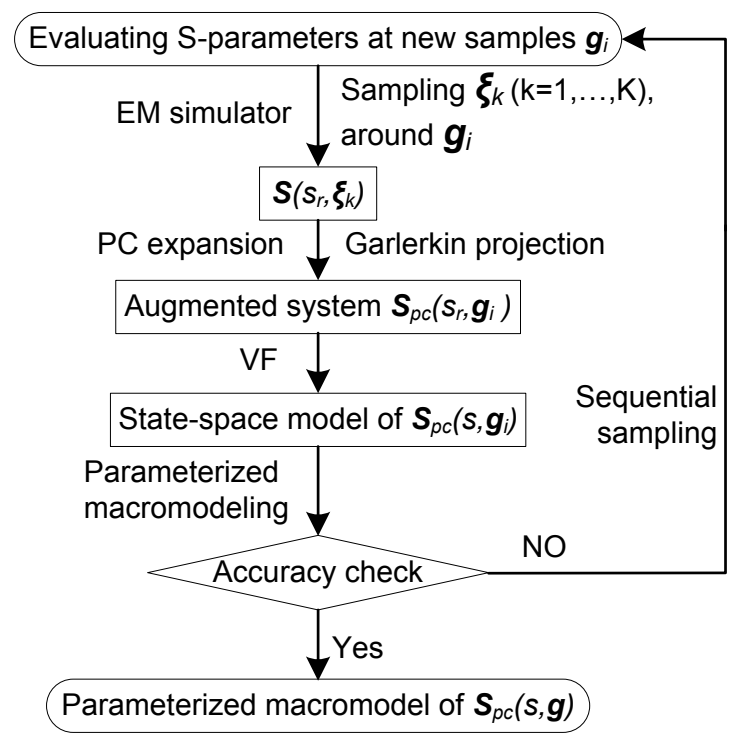

Fig. 1. Flowchart of the proposed parameterized macromodeling technique. 


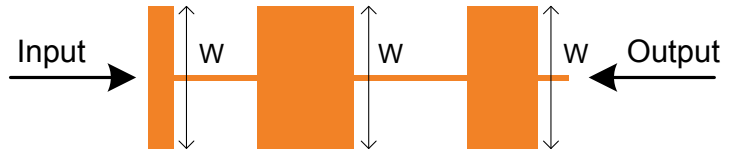

Fig. 2. Geometry of the microstrip lowpass filter under study.

in Section III, since it is always possible to compute the corresponding augmented system [6]. Note that the size of the augmented system depends on the number of the PC basis functions as $N \times(M+1)$. Hence, first accurate $\mathrm{PC}$ models with $M_{i}+1$ basis functions are built separately for different nominal values of the chosen random variable in the design space. Next, the highest $M_{i}$ is chosen to build the corresponding parameterized macromodel $\boldsymbol{S}_{P C}(s, \boldsymbol{g})$ in the entire design space. Note that, the computational cost of building a parameterized macromodel $\boldsymbol{S}_{P C}(s, \boldsymbol{g})$ is similar to the corresponding one for applying the same parameterized macromodeling technique to a deterministic system of size $(M+1) N \times(M+1) N$. However, differently from the deterministic case, $K$ evaluations of the scattering parameters are required to compute the corresponding augmented system for each sample $\boldsymbol{g}_{i}$, where $K$ depends on the particular technique adopted to compute the PC coefficients [6]. Finally, the proposed approach defines a unique framework to model deterministic and stochastic parameters altogether and gives a complete characterization of the system under stochastic effects in the entire design space. However, the downside is that the computational cost to build the parameterized model can be higher than performing the variability analysis (via PCbased approaches, for example) only for the nominal values of interest for the specific problem at hand.

\section{NUMERICAL EXAMPLE}

The microstrip lowpass filter shown in Fig. 2 and described in [14] is analyzed over the frequency range $[1-7] \mathrm{GHz}$. The filter scattering parameters are evaluated in $\mathrm{Matlab}^{1}$ via a quasi-analytical model [15]. The width $W$ of the three microstrips varies in the range $[9-15] \mathrm{mm}$ and is assumed as a deterministic parameter, since the filter performance is not sensitive to its variation caused by the manufacturing tolerances; the height of the substrate $H$ is considered as a Gaussian distributed random variable with nominal values in the range $[1.5-3.5] \mathrm{mm}$ and standard deviation $75 \mu \mathrm{m}$; the relative permittivity of the substrate $\epsilon_{r}$ is a Gaussian random variable with fixed nominal value 4.2 and standard deviation 0.067: once the substrate material is chosen, the only variations of $\epsilon_{r}$ depend on the tolerances of the manufacturing process.

Following the approach described in Section II-B, ten PC basis functions are used to compute the desired model, leading to twenty-port augmented system. In particular, a non-intrusive stochastic testing sampling method [1], [3] is used to minimize the number of samples required for computing the desired PC coefficients. As a result, the augmented system can be built

\footnotetext{
${ }^{1}$ The Mathworks Inc., Natick, MA, USA.
}

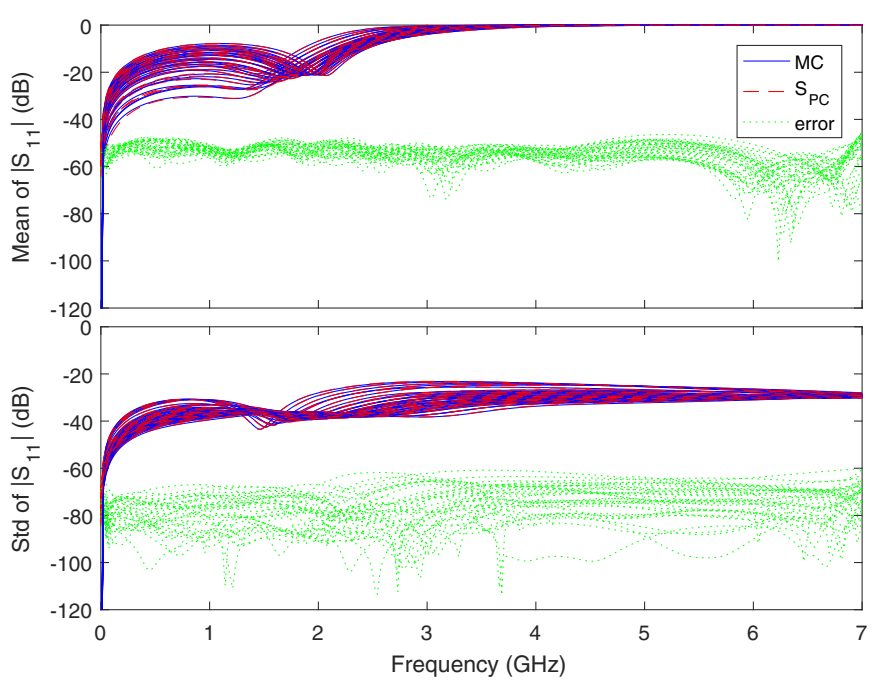

Fig. 3. The mean and standard deviation (std) of the magnitude of $S_{11}(s, \boldsymbol{\xi})$ for 36 nominal values of $\left(W, H, \epsilon_{r}\right)$. The blue lines indicate the MC analysis, the red dash lines are results of the proposed model, while the green dotted lines shows the corresponding absolute error.

by evaluating the scattering parameters at $K=10$ samples of the chosen parameters around each nominal value $\boldsymbol{g}_{i}$. Next, a parameterized macromodel $\boldsymbol{S}_{P C}(s, \boldsymbol{g})$, with $\boldsymbol{g}=\left(W, H, \epsilon_{r}\right)$ is built via the techniques [7], [13] by assuming a maximum absolute error threshold of $-45 \mathrm{~dB}$ between the model response and the computed augmented systems.

Figure 3 shows the modeling accuracy in the frequencydomain: the results of the proposed macromodel for 36 points spreading over the design space (not used to build the model) are compared with the MC analysis performed for 5000 $\left(W, H, \epsilon_{r}\right)$ samples. The accuracy is good for all the points considered.

Time-domain simulations are performed with the following settings: the filter is excited by a source with $50 \Omega$ internal resistance and is terminated on a $50 \Omega$ resistor. The source generates a windowed sinc function voltage pulse with rectangular shape spectrum from DC to $5.2 \mathrm{GHz}$. Note that the parameterized model $\boldsymbol{S}_{P C}(s, \boldsymbol{g})$ is stable and passive: it can be directly applied for time-domain simulations. Six samples equally spaced along each diagonal of the two dimensional design space $(W, H)$ are chosen for accuracy assessment of the model (note that the nominal value of $\epsilon_{r}$ is constant), while the time-domain MC analysis at the same samples is also conducted as a benchmark. In particular, a state-space model is built via the VF algorithm for each sample used in the MC analysis. The corresponding mean and standard deviation of the output voltage are shown in Figs. 4 and 5. It is interesting that all the mean values in Fig. 4 are quite similar, while the corresponding standard deviations present noticeable differences, which indicates that some samples are more robust than others with regard to the variations of the random parameters. Instead, for the points at the other diagonal both the mean and standard deviation are quite sensitive to the parameters variations as Fig. 5 shows. These results show the 

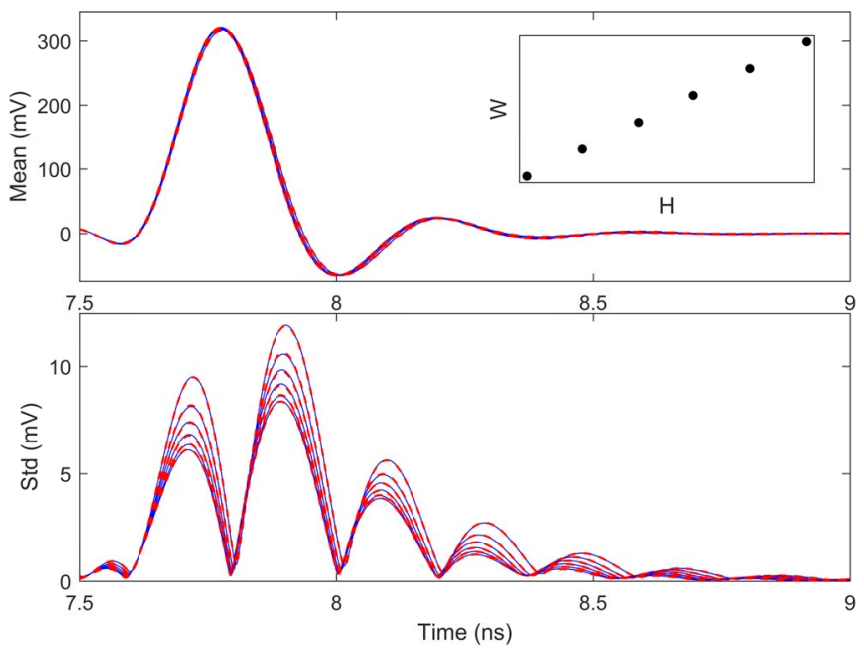

Fig. 4. Mean and standard deviation (std) of the output voltage for six samples equally spaced along one diagonal of the design space: from $(W=9.25, H=1.55) \mathrm{mm}$ to $(W=14.75, H=3.45) \mathrm{mm}$. The blue lines indicate the $\mathrm{MC}$ analysis and red dash lines are obtained via the proposed model.
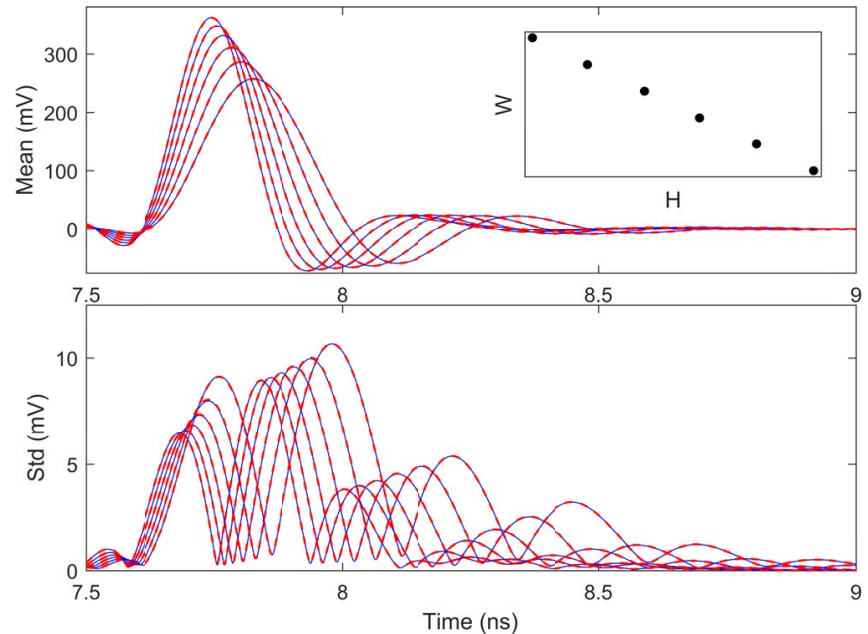

Fig. 5. Mean and standard deviation (std) of the output voltage for six samples equally spaced along one diagonal of the design space: from $(W=14.75, H=1.55) \mathrm{mm}$ to $(W=9.25, H=3.45) \mathrm{mm}$. The blue lines indicate the $\mathrm{MC}$ analysis and red dash lines are obtained via the proposed model.

capability of the proposed method of giving a comprehensive analysis of the filter performance in the entire design space.

Furthermore, complex stochastic moments such as the probability density function (PDF) and cumulative distribution function (CDF) can also be computed via the (inexpensive) sampling of the corresponding PC model in the frequencyand time-domain [6].

The model building process is completed in 9 hours and 29 minutes. However, the frequency- and time-domain variability analysis via the computed model require $2.5 \mathrm{~s}$ and $30 \mathrm{~s}$, respectively, for each nominal value of the parameters considered. Note that a single time-domain MC analysis requires about 4 hours 30 minutes, on average. Hence, the proposed approach is more efficient than the MC method if more than two design points are considered.

\section{CONCLUSion}

A parameterized macromodeling approach for stochastic linear systems is proposed in this contribution. The proposed approach is general, since it can be applied to a large category of systems, and offers a flexible model building phase, where different techniques can be used to compute the proposed macromodel. Furthermore, the proposed technique allows one to perform accurate and efficient frequency- and time-domain variability analysis. A suitable numerical example validates the proposed methodology.

\section{REFERENCES}

[1] Z. Zhang, T. A. El-Moselhy, I. M. Elfadel and L. Daniel, "Stochastic testing method for transistor-level uncertainty quantification based on generalized polynomial chaos," IEEE Trans. Comput.-Aided Design Integr. Circuits Syst, vol. 32, no. 10, pp. 1533-1545, Oct. 2013.

[2] M. R. Rufuie, E. Gad, M. Nakhla and R. Achar, "Generalized hermite polynomial chaos for variability analysis of macromodels embedded in nonlinear circuits," IEEE Trans. Compon., Packag., Manuf. Technol., vol. 4, no. 4, pp. 673-684, Apr. 2014

[3] P. Manfredi and F. G. Canavero, "Efficient statistical simulation of microwave devices via stochastic testing-based circuit equivalents of nonlinear components," IEEE Trans. Microw. Theory Techn., vol. 63 , no. 5, pp. 1502-1511, May 2015.

[4] A. K. Prasad and S. Roy, "Accurate reduced dimensional polynomial chaos for efficient uncertainty quantification of microwave/RF networks," IEEE Trans. Microw. Theory Techn.,, vol. 65, no. 10, pp. 36973708, Oct. 2017.

[5] Y. Ye, D. Spina, P. Manfredi, D. Vande Ginste, and T. Dhaene, "A comprehensive and modular stochastic modeling framework for the variability-aware assessment of signal integrity in high-speed links," IEEE Trans. Electromagn. Compat., vol. 60, no. 2, pp. 459-467, Apr. 2018.

[6] D. Spina, T. Dhaene, L. Knockaert and G. Antonini, "Polynomial chaosbased macromodeling of general linear multiport systems for timedomain analysis," IEEE Trans. Microw. Theory Techn., vol. 65, no. 5, pp. 1422-1433, May 2017.

[7] F. Ferranti, L. Knockaert, T. Dhaene, and G. Antonini, "Parametric macromodeling based on amplitude and frequency scaled systems with guaranteed passivity," Int. J. Numer. Model., vol. 25, no. 2, pp. 139-151, Mar./Apr. 2012.

[8] F. Feng, C. Zhang, J. Ma, and Q. J. Zhang, "Parametric modeling of EM behavior of microwave components using combined neural networks and pole-residue-based transfer functions," IEEE Trans. Microw. Theory Techn., vol. 64, no. 1, pp. 60-77, Jan. 2016.

[9] L. Y. Xiao, W. Shao, T. L. Liang, and B. Z. Wang, "Efficient extreme learning machine with transfer functions for filter design," in 2017 IEEE MTT-S International Microwave Symposium (IMS), Jun. 2017, pp. 555557.

[10] S. Grivet-Talocia, "A perturbation scheme for passivity verification and enforcement of parameterized macromodels," IEEE Trans. Compon., Packag., Manuf. Technol., vol. 7, no. 11, pp. 1869-1881, Nov. 2017.

[11] B. Gustavsen and A. Semlyen, "Rational approximation of frequency domain responses by vector fitting," IEEE Trans. Power Del., vol. 14, no. 3, pp. 1052-1061, Jul. 1999.

[12] D. Deschrijver and T. Dhaene, "Fast passivity enforcement of Sparameter macromodels by pole perturbation," IEEE Trans. Microw. Theory Techn., vol. 57, no. 3, pp. 620-626, Mar. 2009.

[13] K. Chemmangat, F. Ferranti, T. Dhaene, and L. Knockaert, "Scalable models of microwave system responses using sequential sampling on unstructured grids," Int. J. Numer. Model., vol. 27, no. 1, pp. 122-137, Jan./Feb. 2014.

[14] B. Omid and R. Arman, "Design, simulation and construction a low pass microwave filters on the micro strip transmission line," Int. J. Comput. Theory Eng., vol. 4, no. 5, pp. 784-787, Oct. 2012.

[15] K. C. Gupta, R. Garg, I. Bahl, and P. Bhartia, Microstrip Lines and Slotlines, 2nd Edition. Norwood, MA: Artech House, Inc., 1996. 